\title{
An efficient k-means algorithm for the cluster head selection based on SAW and WPM
}

\author{
Anil Khandelwal ${ }^{*}$ and Yogendra Kumar Jain ${ }^{2}$ \\ Research Scholar, Electronics and Communication, Samrat Ashok Technological Institute, Vidisha, MP, India ${ }^{1}$ \\ Professor, Department of Electronics and Instrumentation Engineering, Samrat Ashok Technological Institute, \\ Vidisha, MP, India ${ }^{2}$
}

Received: 15-February-2018; Revised: 28-June-2018; Accepted: 02-July-2018

(C)2018 ACCENTS

\begin{abstract}
A wireless sensor network (WSN) offers the aggregation of data for the communication and processing in the exterior area or the base station. The main purpose of this study was to efficiently select the cluster heads (CHs) and carry out the synchronous data sink operation for the efficient energy and time utilization. An efficient approach based on the $k$-means algorithm for the cluster head selection has been proposed. It also includes simple additive weighting (SAW) and weighted product method (WPM) for the data sink operation priority by the decision performance ranking. In this approach, weights are assigned and pre-processed on the basis of the node operations or the attribute values. These values are used for clustering of the nodes. K-means have been applied for the clustering. The resultant data are then processed with the decision performance ranking methods. We have used SAW and WPM for the selection of CHs from the clusters. The variations in SAW and WPM results are minor and these approaches are efficient in providing the proper CHs selection from the obtained clusters. The result of the random selection priority scale also offers an energy efficient system. The proposed approach results in less delay in packet delivery and offers efficient energy consumption in contrast to the traditional method.
\end{abstract}

Keywords

WSN, CHs, K-means, SAW, WPM.

\section{Introduction}

Wireless sensor networks (WSNs) are used in various applications [1]. The main limitation of WSNs is the limited resources and unreliable communication links [1-3] and there is the need for advanced data mining techniques for data handling is needed as the traditional techniques are not suitable [1-3]. Hence developing an effective data handling techniques for WSNs becomes important. Energy preservation is also a challenging issue in the data management [4]. As it affects the whole network so that some readings cannot be sent to any sink [4]. Therefore handling of data in an efficient manner is needed for the energy preservation. Monitoring of environmental conditions over time and space is also very important factor [5]. The other important factors are cluster heads (CHs) selection, data sink operation and clustering operation on the CHs. Fuzzy decision-making approach is useful for the selection of $\mathrm{CHs}$ which has been proposed by Azad and Sharma [6].

*Author for correspondence
Prominent clustering scheme selection based on unique performance metrics has been suggested by Zeb et al. [7]. Li et al. [8] discussed the data management techniques in sensor networks. It also includes data management systems in sensor networks and in distributed database system differences, the architecture, the data model and the query language, the storing and indexing techniques of sensor data and the operating algorithms, the query processing techniques. Mahmood et al. [9] suggested data mining techniques for extracting knowledge from large continuous arriving data from wireless sensor networks (WSNs). They have provided an extensive survey of the traditional data mining algorithms uses to achieve good performance in a wireless sensor network environment. They have suggested adaptive data mining framework of WSNs for future research because of the limitation in the traditional algorithms. Izadi et al. [10] presented a fuzzy-based data fusion approach for WSN. It is capable of distinguishing and aggregating only true values of the collected data. It reduced the burden of processing the entire data at the base station (BS). 
This also helps in eliminating redundant data and reduces energy consumption and so increases the network lifetime. Anisi et al. [11] suggested cluster and tree structures for data gathering. They have selected most appropriates hops for data forwarding and maximized the lifetime of the whole network. Upadhyay and Mehta [12] focused on delivery latency minimization problem (DLMP). They have presented improved anchor point algorithm with clustering (APAC) in WSNs. They suggested that the latency can be minimized by using better anchor point selection in point substitution method. They have proved that the latency is reduced by point substitution method. Kim and Kim [13] proposed parallel join algorithm. According to the authors it is energy-efficient as the columns are shipped to the join region for final join processing and because of the parallel join processing of sensor data. It improves the performance of the system. Mota et al. [14] proposed network processor architecture. It is used to handle information at sensor network base stations. It optimizes information processing and the magnitude faster than an architecture based on traditional processor. Tian et al. [15] presented a sink node design. It contains the power module, the storage/display module and the communication module. Zigbee, GPRS and Ethernet techniques are used. The sink node is capable of bridging the user's terminal with sensor nodes for information transmission. Rhee and Liu [16] suggested persistent dynamic routing. It provides high data reliability needed for mission-critical networks. The results obtained are much higher scalability and throughput without sacrificing reliability. Palpanas [17] review and analyses WSNs problems as real-time collection of the sensed data, and real-time processing of these data series. Based on these aspects different methods are discussed with their advantages and flaws. Bergelt et al. [1] proposed a strategy of aggregation to tie technological prerequisites for efficient use of energy and the handling of large data volumes. They have demonstrated with the experimental energy conservation potential based with actual sensor platforms. Ghayvat et al. [18] reported a new protocol especially developed to address smart homes for assisted living. The developed protocol has been used in an old home built in 1938, which was converted into a smart home with the use of sensing technologies.

The main objectives of this study are as follows:

- Applying the k-means algorithm for the selection of CHs.
- To provide a random selection of $\mathrm{CHs}$ based on the operations and data handling mechanism specified by the data sink.

- To determine the ranking of $\mathrm{CHs}$ for the selection.

- To determine the data sink operation priority by simple additive weighting (SAW) and weighted product method (WPM).

- A comparative study based on the SAW and WPM has been analyzed.

\section{Methods}

This framework has been developed on the Java platform version 7. Framework designing and execution have been performed on NetBeans IDE 7.2 environment. In the proposed approach IN and OUT nodes are considered for the communication pathway for the sink node. We have proposed an efficient approach for the $\mathrm{CHs}$ selection with the confirmation of the packet delivery for all. In the first phase the data pre-processing is applied based on the weight assigned. It is assigned based on the node's operations. There are five data sink attributes. These are read $(\mathrm{R})$, write $(\mathrm{W})$, update $(\mathrm{U})$, send $(\mathrm{S})$ and receive $(\mathrm{Re})$. The priority values for these attributes are assigned by Java random class. The range of these values lies between 1 to 10 . It reduces automatically based on the computation time in the data collection and sending to the sink node. It is used for the clustering purpose. In this phase the data have been converted into the computational phase for different mathematical calculation and for the feasibility study.

The second phase is the clustering phase. This approach has the capability of selection of appropriate group based on k-means (Figure 1). Kmeans is efficient and simple clustering algorithm [19]. Because of the better clustering capabilities, it is used in different research areas [20]. K-means have been applied in the pre-processed data obtained from the first phase. The group is selected based on the weight aggregation of the attribute values in our approach. The processes of operations are completely random, so that there is no biasness. Algorithm 1shows the k-means algorithm which is used to cluster the nodes based on the weight for the $\mathrm{CHs}$ selection. The main benefit of k-means algorithm is that it is computationally faster if the number of clusters is small and produce tighter cluster [19, 20]. After clustering, cluster group is selected based on the maximum weight strength of the cluster. Ranking algorithms have been applied in this phase. Then in the cluster, $\mathrm{CHs}$ are selected based on the ranking 
strategy combined with our approach. Multiplecriteria decision-making (MCDM) is an approach for combining decision performances [21]. It is used in several areas of energy efficiency [21,22]. The main methods of MCDM are SAW, WPM, analytical hierarchy process (AHP), the technique for the order of preference by similarity to the ideal solution (TOPSIS), etc. We have used SAW and WPM in our approach for the decision performance ranking. SAW is a simple and widely used scoring method based on a weighted linear combination [23]. In this approach weighted average have been used with the priority values. For each alternative an evaluation score is calculated. It is multiplying the priority value or the scaled value provided to the respective attribute. Then add all the obtained multiplicative value for the individual node attributes. It provides the score for the individuals which can be helpful in the ranking criteria. The benefit of this technique is that it is a corresponding direct change of the crude information which implies that the relative request of size of the institutionalized scores stays break even with. WPM is the variant of SAW [24, 25].
The main computational difference is in the mathematical process. In this approach multiplication is applied instead of the addition operation. The alternatives used also compared with the others. It is compared by multiplying a number of ratios. This is assigned to the each criterion available in the node. The same is applicable as the power equivalent of the relative weight to the corresponding criterion [24, 25]. This dispenses with any units of measure and accordingly takes into account dimensionless investigation. So the strategy can be utilized as a part of single-and multi-dimensional choice issues. In our case the priority values are considered as 0.625 , 0.125 and 0.25 .

Data transmission is the main part or task in nodes communication [26]. In the packet transfer process the energy consumption is in proportion to the distance [26]. As in our approach the distance (d) is same and differentiated with an unbiased approach. So the energy consumed is the time consumed in the transfer process for the $\mathrm{x}$ bit data.

$E=d^{2} x$

\section{Algorithm 1: K-means algorithm}

$\mathrm{C}=\left\{\mathrm{c}_{1}, \mathrm{c}_{2}, \mathrm{c}_{3} \ldots \ldots \ldots \ldots \ldots \ldots \ldots \ldots \mathrm{c}_{\mathrm{n}}\right\}$ be the set of centroids.

$\mathrm{D}=\left\{\mathrm{d}_{1}, \mathrm{~d}_{2}, \mathrm{~d}_{3} \ldots \ldots \ldots \ldots \ldots \ldots \ldots \mathrm{d}_{\mathrm{n}}\right\}$ be the set of data points.

Step 1: Initialize the centroids randomly.

Step 2: Each data point is assigned to a cluster based on data point and cluster center distance. Euclidean distance is used for the distance measurement.

$$
\begin{aligned}
& \mathrm{J}(\mathrm{c})=\sum_{j=1}^{k} \sum_{i=1}^{n}\left\|d_{i}^{(j)}-c_{j}\right\|^{2} \\
& \mathrm{~d}_{\mathrm{i}}-\mathrm{c}_{\mathrm{j}} \text { is the Euclidean distance. }
\end{aligned}
$$

Where $\mathrm{k}$ is the number of cluster and $\mathrm{n}$ is the number of cases

Step 3: Minimum distance is preferred in data point assignment.

Step 4: Then new cluster center is recalculated

$$
\mathrm{c}_{\mathrm{i}}=\left(\frac{1}{n_{i}}\right) \sum_{j=1}^{n_{i}} d_{i}
$$

Step 5: The process is stopped after the reassignment.

\section{Algorithm 2: SAW method}

Step 1: Normalize the attribute values present in the decision matrix.

Step 2: Score is evaluated for the alternatives

$$
\mathrm{S}_{\mathrm{i}}=\sum_{j=1}^{n} w_{j} r_{i j}
$$

$\mathrm{S}_{\mathrm{i}}$ is the complete score of the $i^{\text {th }}$ alternative

$\mathrm{W}_{\mathrm{j}}$ is the weight of $\mathrm{j}^{\text {th }}$ criterion

Step 3: The score is calculated for each alternatives.

\section{Algorithm 3: WPM}

Step 1: Normalize the attribute values present in the decision matrix.

Step 2: Score is evaluated for the alternatives

$$
\mathrm{R}\left(\frac{A_{k}}{A_{l}}\right)=\prod_{j=1}^{n}\left(\frac{a_{k j}}{a_{i j}}\right)^{w_{j}}
$$

$a_{i j}$ is the score of the $i^{\text {th }}$ alternative in the $j^{\text {th }}$ criterion and $w_{j}$ is the weight of the $j$-th criterion

Step 3: The score is calculated for each alternatives. 
Khandelwal and Jain

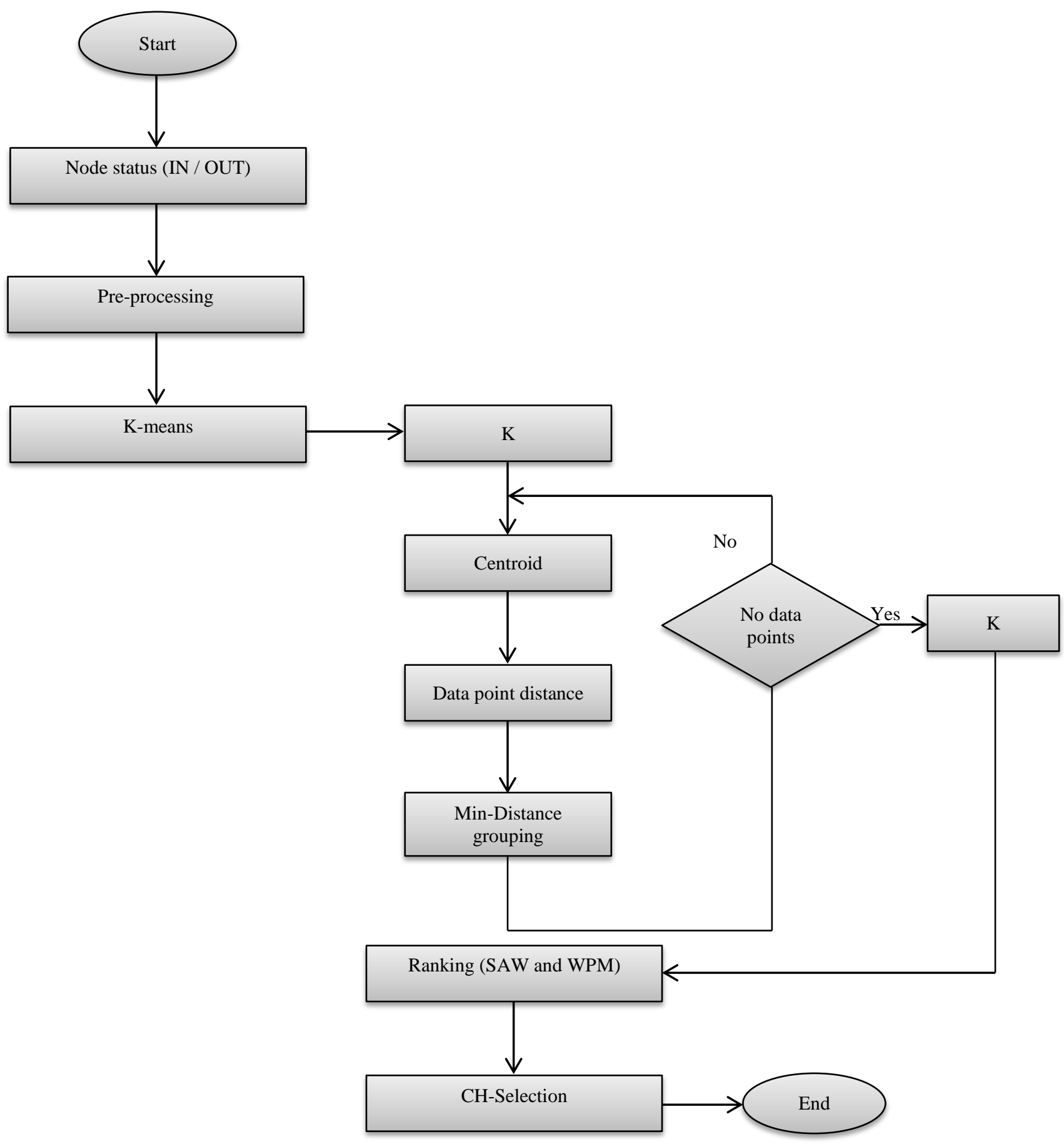

Figure 1 Flowchart of complete working mechanism

\section{Results}

In this section, the results obtained from the proposed approach have been discussed and compared with the other existing techniques. In order to carry out the experiment, 50 nodes are considered and for comparison 100, 150, 200, 250 and 300 nodes are considered. Table 1 shows the initial attribute weight values. The range of these values lies between 1 and 10 , which automatically reduces depending on the computation time in the data collection and sending the same to the sink node. It is used for the clustering purpose. We obtain Tables 2 and 3 after clustering. 
The total attribute sum for cluster 1 is 178 and for cluster 2 is 252 . Thus, cluster 2 will be selected. The $\mathrm{CHs}$ are selected from cluster 2 and from cluster 2, $\mathrm{CHs}$ are selected according to the ranking approach. First random selection priority scale has been assigned from $\{1,2,3\},\{1\},\{2,3\},\{2,3,4\},\{4,5\}$ and $\{5\}$. It is 1 for R, 2 for $W, 3$ for $U, 4$ for $S$ and 5 for Re. It means only those operations that are assigned to the concern nodes are performed. It may help in the energy consumption. It is shown in Tables 4 and 5. SAW and WPM methods are applied to rank CHs for the selection of the data transportation and other operations are performed. Tables 6 and 7 show the initial score for the initial sink attributes. Figure 2 shows the SAW values obtained from cluster 1 .

Figure 3 shows the SAW values obtained from cluster 2. It clearly shows the ranking of $\mathrm{CHs}$ from cluster 1 and 2 (SAW). Figure 4 shows the WPM values obtained from cluster 1. Figure 5 shows the WPM values obtained from cluster 2 . It clearly shows the ranking of CHs from cluster 1 and 2 (WPM). It is clear from Figures 2-5 that the variations in the outcome of the SAW and WPM are minor. Both algorithms suggest the same selection in our case. Table 8 shows the packet delivery time in cluster 1 (cycle 1). Status 1, 2 and 3 show that the priority scale time, assigned randomly. Table 9 shows the packet delivery time in cluster 2 (cycle 1). Table 10 shows the packet delivery time in cluster 1 (cycle 2 ). Table 11 shows the packet delivery time in cluster 1 (cycle 2). The time taken is given in milliseconds. Figure 6 shows the packet delivery time in cluster 1 for the SAW method. Figure 7 shows the packet delivery time in cluster 1 (aggregate operations) for the SAW method. Figure 8 shows the packet delivery time in cluster 1 for the SAW method. Figure 9 shows the packet delivery time in cluster 1 (aggregate operations) for the SAW method. Figure 10 shows the time in seconds for the packet delivery comparison from the traditional method. Figure 11 shows the packet transmission energy comparison from the traditional method.

Table 1 Initial attributes weight

\begin{tabular}{|c|c|c|c|c|c|}
\hline Node & $\mathbf{R}$ & $\mathbf{W}$ & $\mathbf{U}$ & $\mathbf{S}$ & RE \\
\hline Node 1 & 0 & 2 & 6 & 1 & 1 \\
\hline Node 2 & 5 & 7 & 7 & 6 & 3 \\
\hline Node 5 & 0 & 0 & 8 & 2 & 4 \\
\hline Node 7 & 0 & 5 & 5 & 5 & 4 \\
\hline Node 10 & 3 & 8 & 8 & 2 & 2 \\
\hline Node 11 & 6 & 4 & 9 & 8 & 1 \\
\hline Node 14 & 3 & 8 & 8 & 7 & 4 \\
\hline Node 16 & 4 & 2 & 8 & 7 & 7 \\
\hline Node 18 & 6 & 1 & 6 & 2 & 6 \\
\hline Node 19 & 8 & 2 & 3 & 6 & 8 \\
\hline Node 20 & 1 & 9 & 1 & 3 & 3 \\
\hline Node 22 & 6 & 8 & 6 & 9 & 7 \\
\hline Node 26 & 1 & 1 & 5 & 8 & 1 \\
\hline Node 27 & 5 & 7 & 8 & 9 & 2 \\
\hline Node 28 & 8 & 4 & 2 & 9 & 1 \\
\hline Node 31 & 2 & 5 & 9 & 4 & 2 \\
\hline Node 34 & 7 & 3 & 9 & 2 & 2 \\
\hline Node 36 & 9 & 9 & 2 & 8 & 5 \\
\hline Node 41 & 6 & 5 & 6 & 1 & 9 \\
\hline Node 42 & 1 & 9 & 7 & 8 & 8 \\
\hline Node 47 & 6 & 4 & 4 & 7 & 3 \\
\hline
\end{tabular}

Table 2 Cluster 1

\begin{tabular}{|c|c|c|c|c|c|c|}
\hline Node & $\mathbf{R}$ & $\mathbf{W}$ & $\mathbf{U}$ & $\mathbf{S}$ & RE & Total \\
\hline Node 1 & 0 & 2 & 6 & 1 & 1 & 10 \\
\hline Node 5 & 0 & 0 & 8 & 2 & 4 & 14 \\
\hline Node 10 & 3 & 8 & 8 & 2 & 2 & 23 \\
\hline Node 14 & 3 & 8 & 8 & 7 & 4 & 30 \\
\hline Node 18 & 6 & 1 & 6 & 2 & 6 & 21 \\
\hline Node 20 & 1 & 9 & 1 & 3 & 3 & 17 \\
\hline Node 26 & 1 & 1 & 5 & 8 & 1 & 16 \\
\hline Node 28 & 8 & 4 & 2 & 9 & 1 & 24 \\
\hline Node 34 & 7 & 3 & 9 & 2 & 2 & 23 \\
\hline
\end{tabular}


Khandelwal and Jain

Table 3 Cluster 2

\begin{tabular}{lllllll}
\hline Node & R & W & U & S & RE & Total \\
\hline Node 2 & 5 & 7 & 7 & 6 & 3 & 28 \\
Node 7 & 0 & 5 & 5 & 5 & 4 & 19 \\
Node 11 & 6 & 4 & 9 & 8 & 1 & 28 \\
Node 16 & 4 & 2 & 8 & 7 & 7 & 28 \\
Node 19 & 8 & 2 & 3 & 6 & 8 & 27 \\
Node 22 & 6 & 8 & 6 & 9 & 7 & 36 \\
Node 27 & 5 & 7 & 8 & 9 & 2 & 31 \\
Node 31 & 2 & 5 & 9 & 4 & 2 & 22 \\
Node 36 & 9 & 9 & 2 & 8 & 5 & 33 \\
\hline
\end{tabular}

Table 4 Set status 1

\begin{tabular}{lllll}
\hline Node & Status & Status1 & Status2 & Status3 \\
\hline Node 1 & $1,2,3$ & 5 & 10 & 6 \\
Node 5 & $1,2,3$ & 5 & 10 & 6 \\
Node 10 & 2,3 & 10 & 6 & 0.1 \\
Node 14 & $2,3,4$ & 10 & 6 & 5 \\
Node 18 & 5 & 5 & 0.1 & 0.1 \\
Node 20 & 2,3 & 10 & 6 & 0.1 \\
Node 26 & 4,5 & 5 & 5 & 0.1 \\
Node 28 & 1 & 5 & 0.1 & 0.1 \\
Node 34 & $1,2,3$ & 5 & 10 & 6 \\
\hline
\end{tabular}

Table 5 Set status 2

\begin{tabular}{lllll}
\hline Node & Status & Status1 & Status2 & Status3 \\
\hline Node 2 & $2,3,4$ & 10 & 6 & 5 \\
Node 7 & 2,3 & 10 & 6 & 0.1 \\
Node 11 & 1 & 5 & 0.1 & 0.1 \\
Node 16 & 4,5 & 5 & 5 & 0.1 \\
Node 19 & 2,3 & 10 & 6 & 0.1 \\
Node 22 & 1 & 5 & 0.1 & 0.1 \\
Node 27 & 1 & 5 & 0.1 & 0.1 \\
Node 31 & 2,3 & 10 & 6 & 0.1 \\
Node 36 & $1,2,3$ & 5 & 10 & 6 \\
\hline
\end{tabular}

Table 6 Score 1

\begin{tabular}{llll}
\hline Node & Status & Status1 & Status2 \\
\hline Node 1 & 1 & 0.01 & $1.66666666666667 \mathrm{E}-02$ \\
Node 5 & 1 & 0.01 & $1.66666666666667 \mathrm{E}-02$ \\
Node 10 & 0.5 & $1.66666666666667 \mathrm{E}-02$ & 1 \\
Node 14 & 0.5 & $1.66666666666667 \mathrm{E}-02$ & 0.02 \\
Node 18 & 1 & 1 & 1 \\
Node 20 & 0.5 & $1.66666666666667 \mathrm{E}-02$ & 1 \\
Node 26 & 1 & 0.02 & 1 \\
Node 28 & 1 & 1 & 1 \\
Node 34 & 1 & 0.01 & $1.66666666666667 \mathrm{E}-02$ \\
\hline
\end{tabular}

Table 7 Score 2

\begin{tabular}{llll}
\hline Node & Status & Status1 & Status2 \\
\hline Node 2 & 0.5 & $1.66666666666667 \mathrm{E}-02$ & 0.02 \\
Node 7 & 0.5 & $1.66666666666667 \mathrm{E}-02$ & 1 \\
Node 11 & 1 & 1 & 1 \\
Node 16 & 1 & 0.02 & 1 \\
Node 19 & 0.5 & $1.66666666666667 \mathrm{E}-02$ & 1 \\
Node 22 & 1 & 1 & 1 \\
Node 27 & 1 & 1 & 1 \\
Node 31 & 0.5 & $1.66666666666667 \mathrm{E}-02$ & 1 \\
Node 36 & 1 & 0.01 & $1.66666666666667 \mathrm{E}-02$ \\
\hline 196 & & &
\end{tabular}


International Journal of Advanced Computer Research, Vol 8(37)

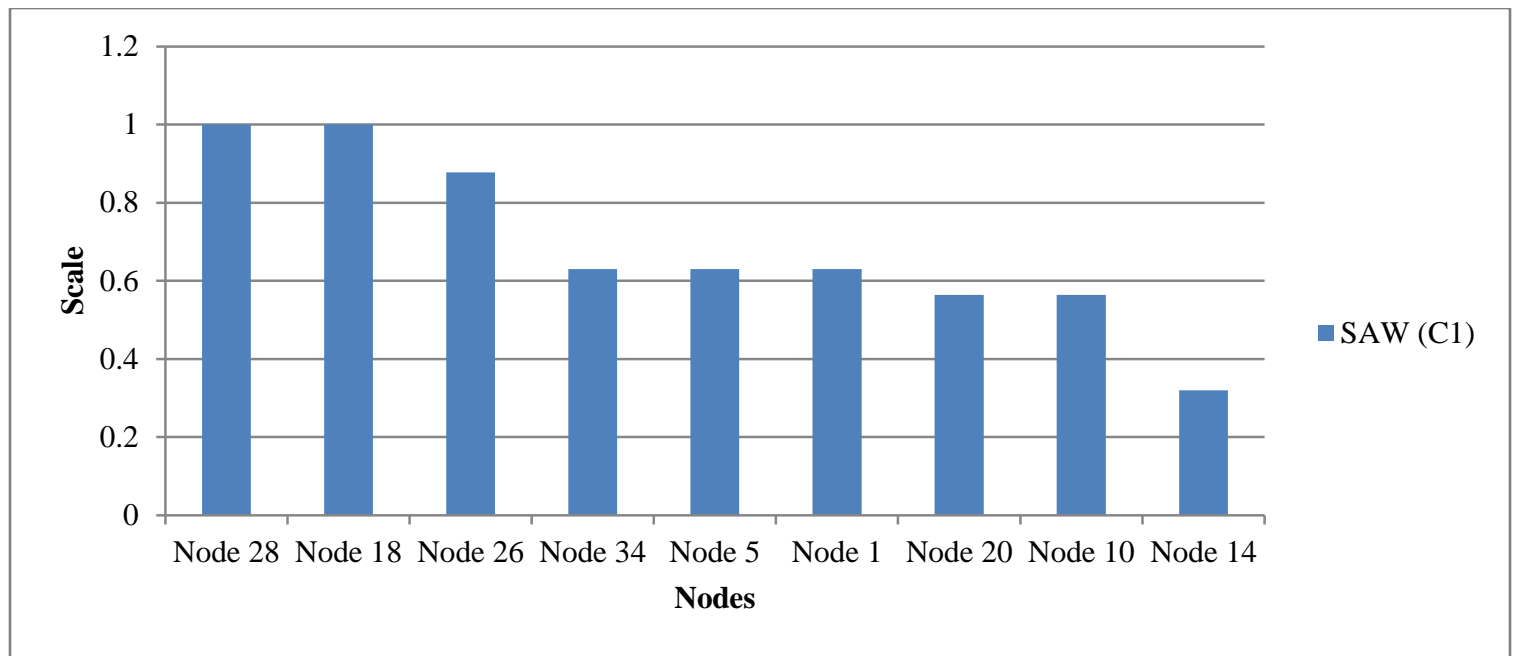

Figure 2 SAW values obtained for the cluster 1

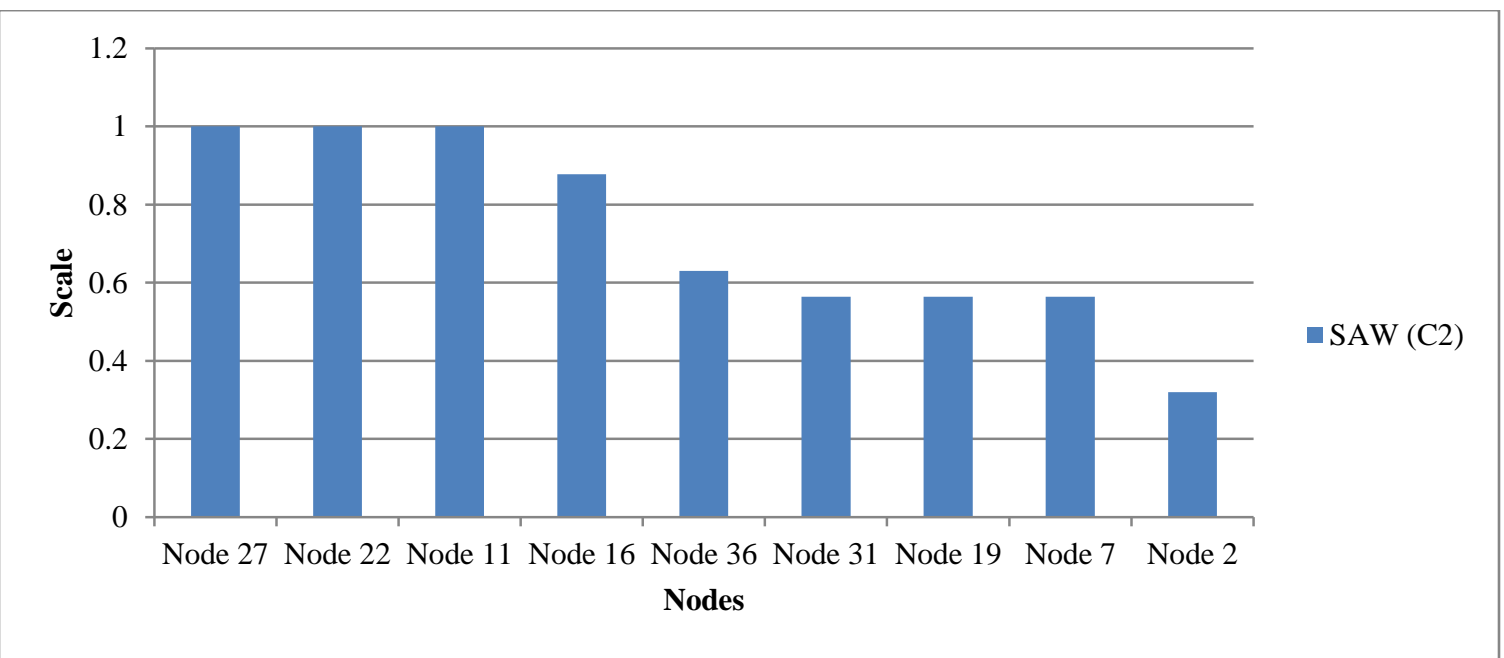

Figure 3 SAW values obtained for the cluster 2

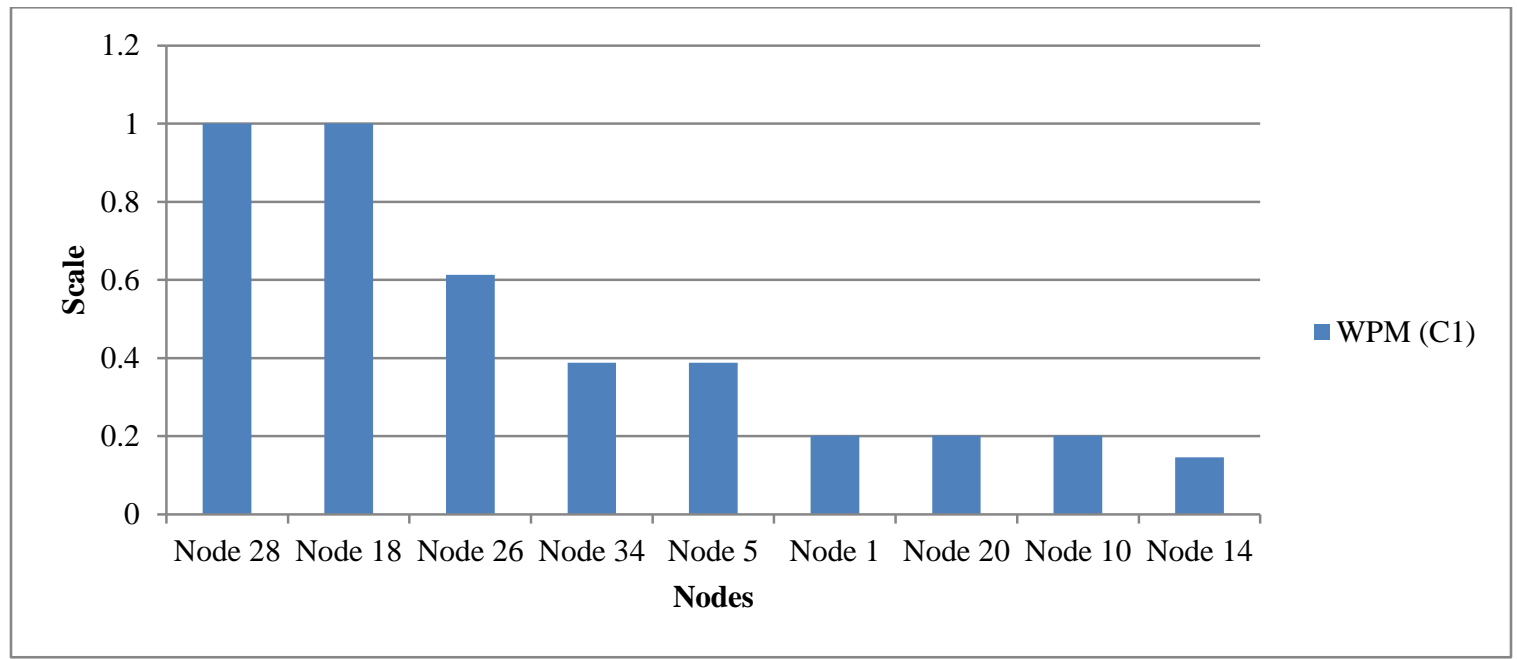

Figure 4 WPM values obtained for the cluster 1

197 
Khandelwal and Jain

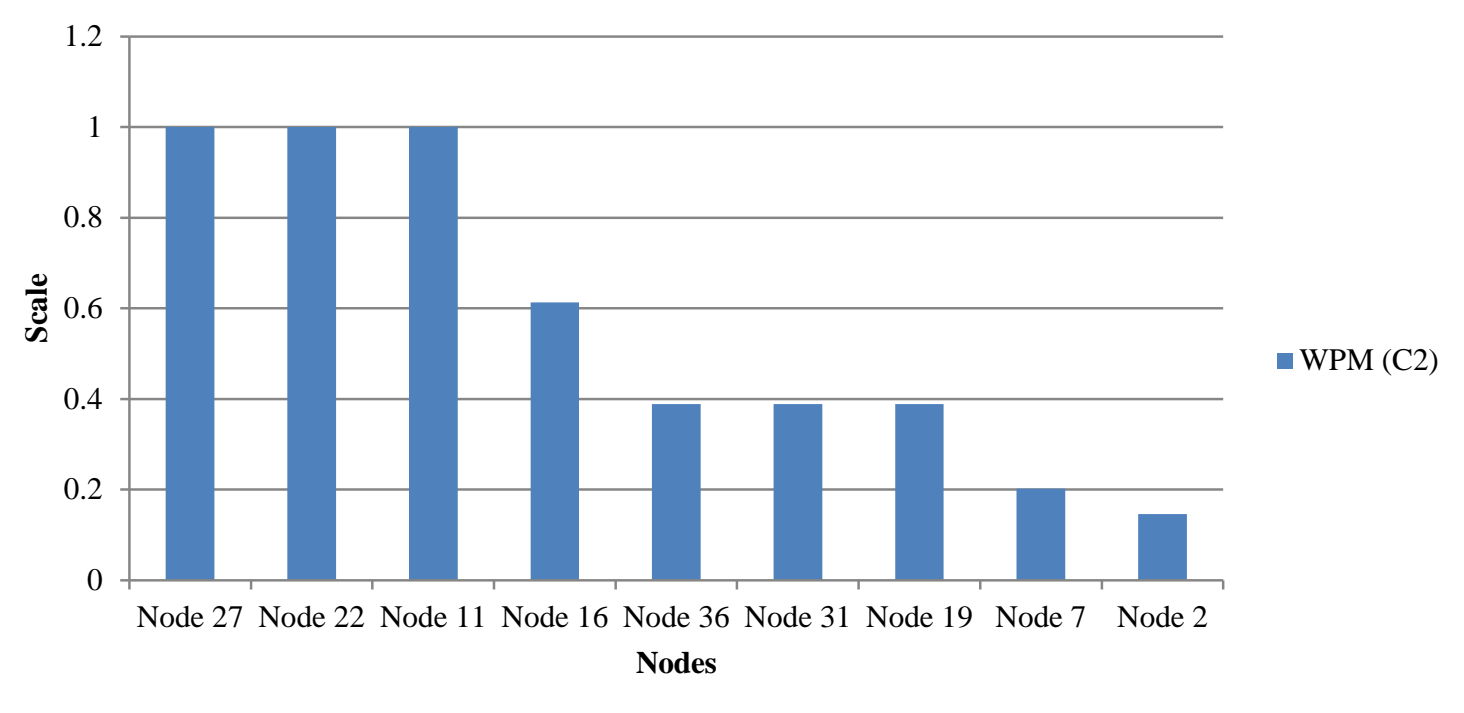

Figure 5 WPM values obtained for the cluster 2

Table 8 Packet delivery time in cluster 1 (cycle 1)

\begin{tabular}{llll}
\hline Node & Status1 & Status2 & Status 3 \\
\hline Node 28 & 145 & 0 & 0 \\
Node 18 & 6 & 0 & 0 \\
Node 26 & 16 & 4 & 0 \\
Node 34 & 72 & 1 & 1590 \\
Node 5 & 71 & 1 & 1512 \\
Node 1 & 69 & 0 & 1538 \\
Node 20 & 0 & 1521 & 0 \\
Node 10 & 1 & 1537 & 0 \\
Node 14 & 1 & 1519 & 0 \\
\hline
\end{tabular}

Table 9 Packet delivery time in cluster 2 (cycle 1 )

\begin{tabular}{llll}
\hline Node & Status1 & Status2 & Status3 \\
\hline Node 27 & 89 & 0 & 0 \\
Node 22 & 85 & 0 & 0 \\
Node 11 & 103 & 0 & 0 \\
Node 16 & 0 & 3 & 0 \\
Node 36 & 77 & 0 & 1547 \\
Node 31 & 0 & 1553 & 0 \\
Node 19 & 0 & 1564 & 0 \\
Node 7 & 0 & 1567 & 0 \\
Node 2 & 0 & 1554 & 0 \\
\hline
\end{tabular}

Table 10 Packet delivery time in cluster 1 (cycle 2)

\begin{tabular}{llll}
\hline Node & Status1 & Status2 & Status3 \\
\hline Node 28 & 79 & 0 & 0 \\
Node 18 & 3 & 0 & 0 \\
Node 26 & 0 & 3 & 0 \\
Node 20 & 1 & 1556 & 0 \\
Node 10 & 0 & 1533 & 0 \\
Node 34 & 70 & 0 & 1611 \\
Node 5 & 77 & 0 & 1555 \\
Node 1 & 97 & 0 & 1553 \\
Node 14 & 0 & 1545 & 1 \\
\hline
\end{tabular}


Table 11Packet delivery time in cluster 2 (cycle 2)

\begin{tabular}{llll}
\hline Node & Status1 & Status2 & Status3 \\
\hline Node 27 & 77 & 0 & 0 \\
Node 22 & 100 & 0 & 0 \\
Node 11 & 97 & 0 & 0 \\
Node 16 & 0 & 3 & 0 \\
Node 31 & 0 & 1569 & 0 \\
Node 19 & 1 & 1563 & 0 \\
Node 7 & 1 & 1574 & 0 \\
Node 36 & 72 & 0 & 1564 \\
Node 2 & 0 & 1595 & 1 \\
\hline
\end{tabular}

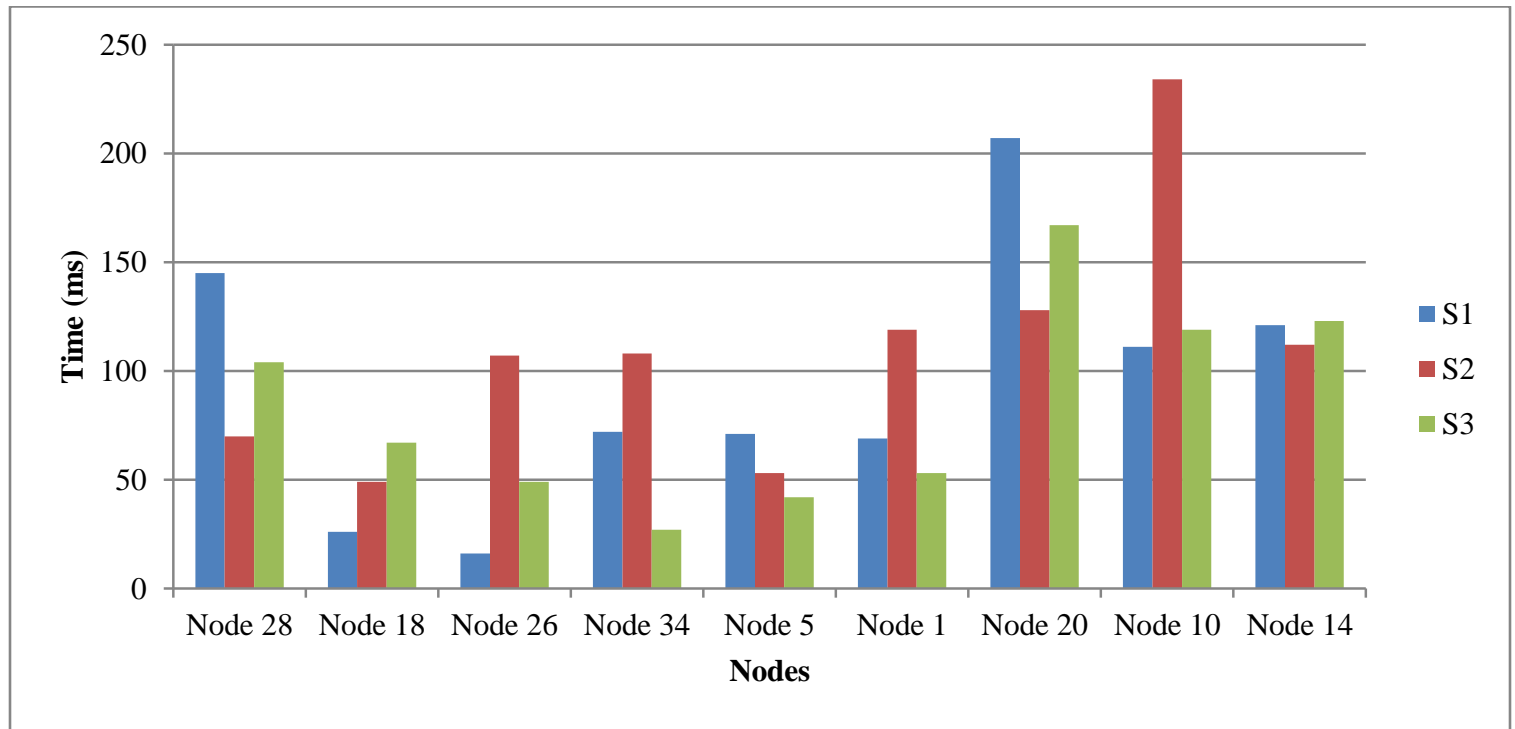

Figure 6 Packet delivery time in cluster 1 for the SAW method

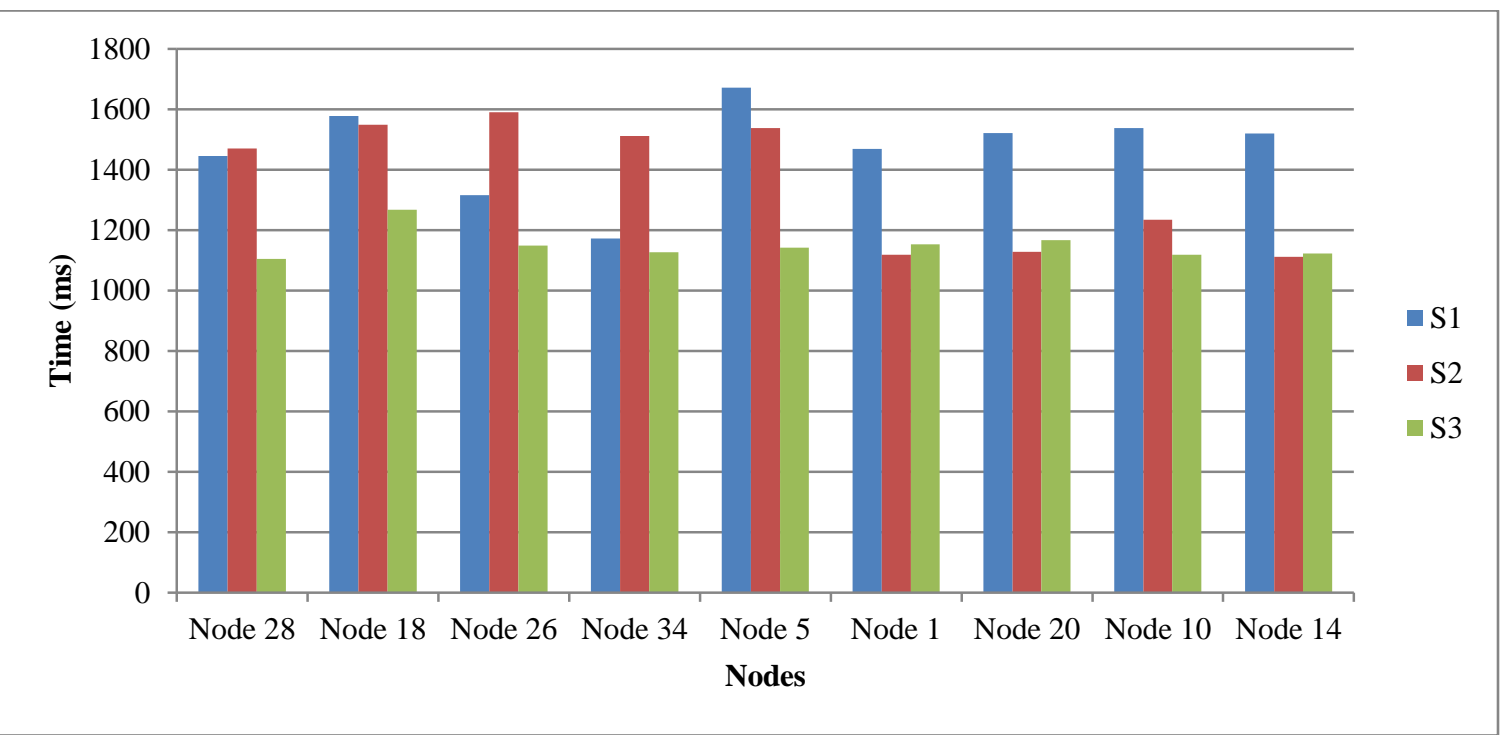

Figure 7 Packet delivery time in cluster 1 (aggregate operations) for the SAW method 
Khandelwal and Jain

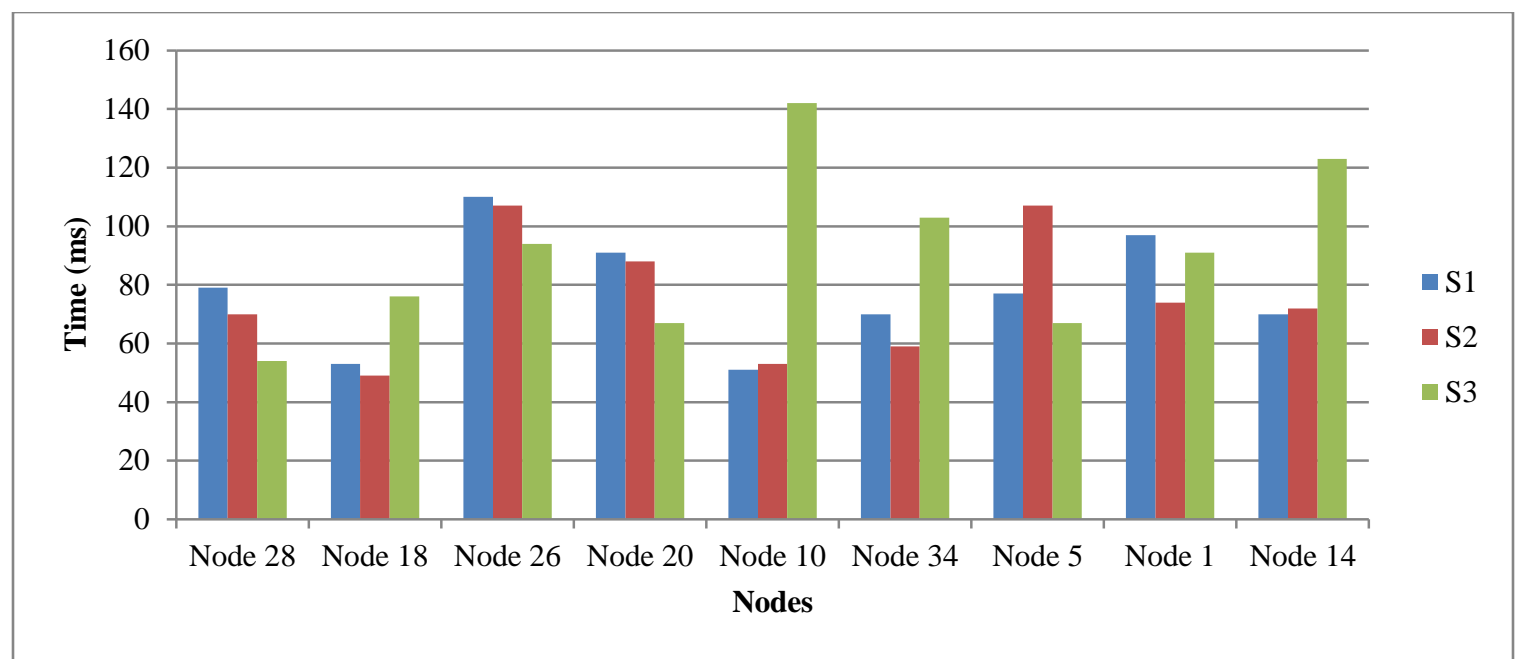

Figure 8 Packet delivery time in cluster 1 for the WPM method

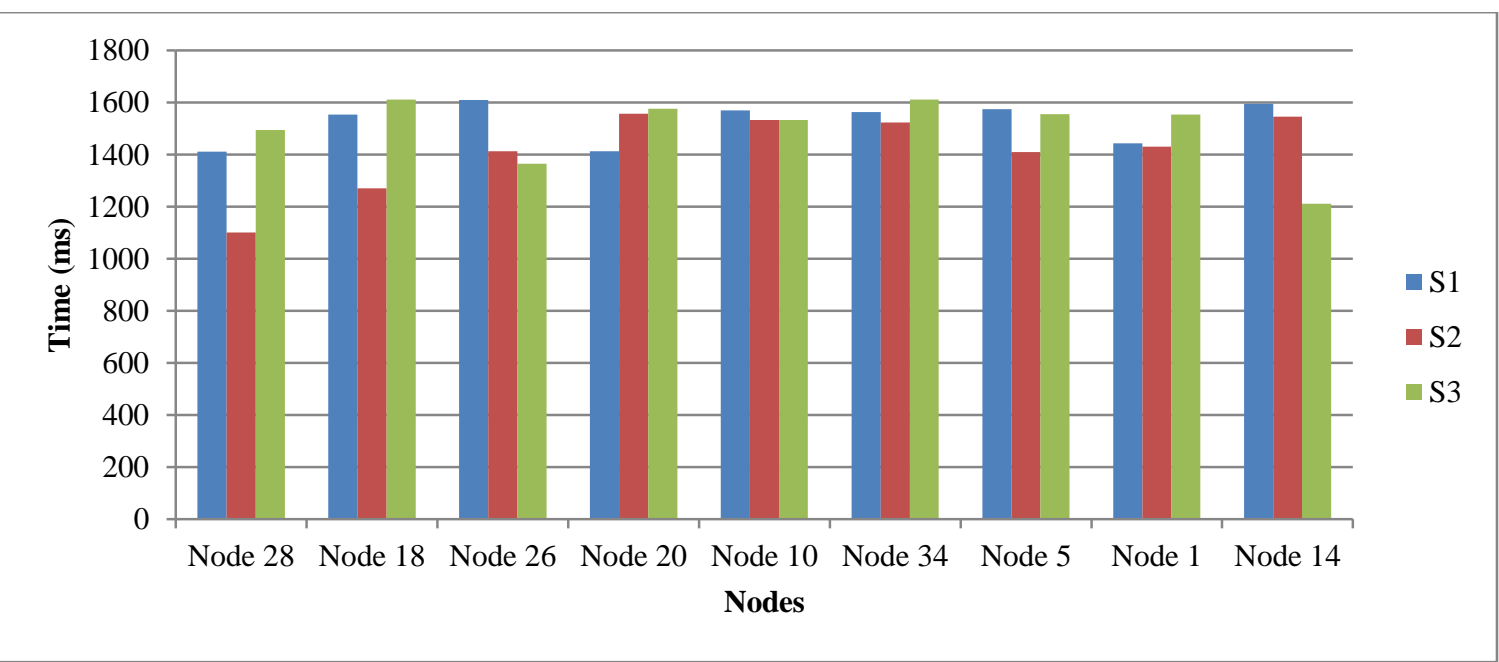

Figure 9 Packet delivery time in cluster 1 (aggregate operations) for the WPM method

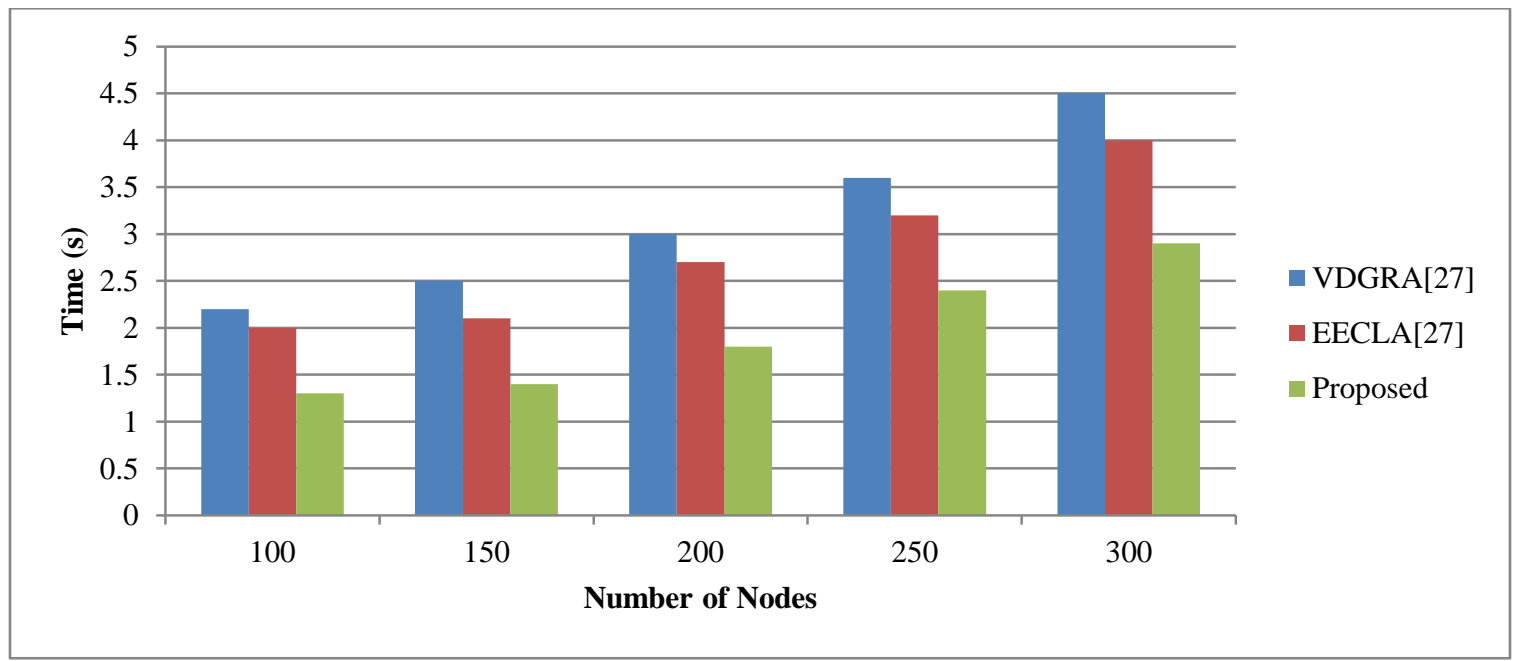

Figure 10 Time in packet delivery 


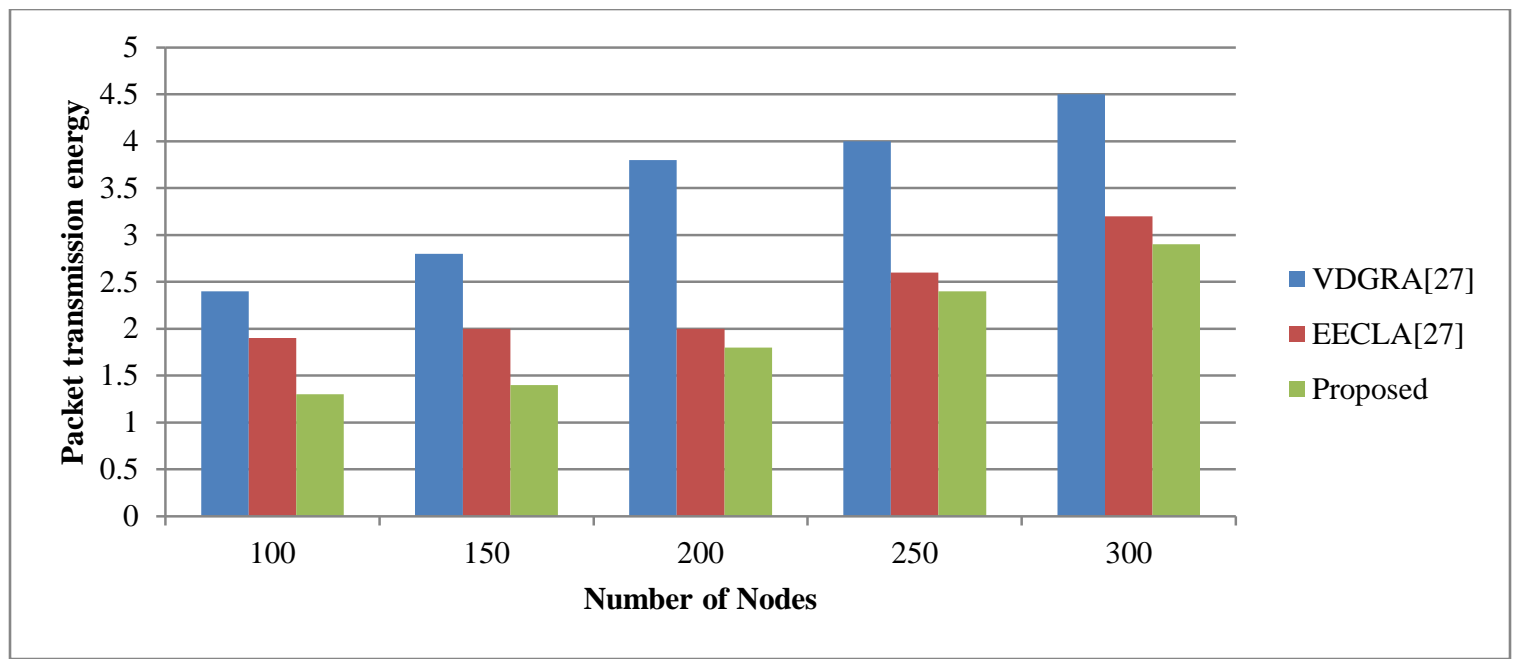

Figure 11 Packet transmission energy consumption

\section{Discussion}

The presented approach shows the capability of clustering the nodes of similar nature based on the attributes' weight. It is capable of ranking through SAW and WPM methods for the selection of CHs in the cluster. The results clearly indicate that the SAW and WPM are efficient in the appropriate $\mathrm{CHs}$ selection and the distribution of the operation so that the energy utilization is minimized. There are minor variations in the outcome of the SAW and WPM, which is the indication of correct and standard ranking. By this, it can be asserted that the proposed approach is capable in short packet delivery time or the delay in delivery is less and offers efficient energy consumption compared to the traditional method. The scales have been considered for the individual and aggregate attributes for the $\mathrm{CHs}$, which outperform in all the cases. We compared the approach with the methods of Nagamalar and Rangaswamy [27]. They considered 100 nodes with a header size of 25 bytes and the data size of 500 bytes. In our approach, the size and other things are dynamic and change at runtime. But for comparison, we also considered 100 nodes with a packet send size of 19405 bytes and the received size of 730 bytes, which is larger. The proposed methods have been compared to the virtual grid-based dynamic routes adjustment (VGDRA) and energy efficient clustering and localization algorithms (EECLA). Our approach with dynamic scaling outperforms in both packet delivery and energy consumption.

\section{Conclusion}

In this paper, a data clustering approach along with decision performance ranking method has been applied for the proper selection of $\mathrm{CHs}$ and synchronization in the sink for the communication environment. K-means algorithm has been applied for the clustering. SAW and WPM methods have been applied for the decision performance ranking. The capability of clustering the nodes has been considered through the attributes' weight and then the ranking method is applied to the appropriate selection in the clusters. A total of 50 nodes have been considered for the experimentation. For comparison, different variations have been considered. The results show that the presented approach outperforms in terms of $\mathrm{CHs}$ selection, operation distribution, packet delivery time, and energy consumption.

\section{Acknowledgment}

None.

\section{Conflicts of interest}

The authors have no conflicts of interest to declare.

\section{References}

[1] Bergelt R, Vodel M, Hardt W. Energy efficient handling of big data in embedded, wireless sensor networks. In sensors applications symposium 2014 (pp. 53-8). IEEE.

[2] Vodel M, Hardt W. Data aggregation in resourcelimited wireless communication environmentsdifferences between theory and praxis. In international conference on control, automation and information sciences 2012 (pp. 208-13). IEEE.

[3] Sarma HK. Grid based data gathering in multi-channel wireless sensor network. In international conference on information technology 2016 (pp. 114-7). IEEE.

[4] Hung CC, Hsieh CC. Big data management on wireless sensor networks. In big data analytics for sensor-network collected intelligence 2017 (pp. 99116). 
[5] Roadknight C, Parrott L, Boyd N, Marshall IW. Realtime data management on a wireless sensor network. International Journal of Distributed Sensor Networks. 2005; 1(2):215-25.

[6] Azad P, Sharma V. Cluster head selection in wireless sensor networks under fuzzy environment. ISRN Sensor Networks. 2013:1-8.

[7] Zeb A, Islam AM, Zareei M, Al Mamoon I, Mansoor $\mathrm{N}$, Baharun S, et al. Clustering analysis in wireless sensor networks: the ambit of performance metrics and schemes taxonomy. International Journal of Distributed Sensor Networks. 2016; 12(7): 1-24.

[8] Li J, Cai Z, Li J. Data management in sensor networks. Wireless Sensor Networks and Applications. Signals and Communication Technology. 2008. Springer.

[9] Mahmood A, Shi K, Khatoon S, Xiao M. Data mining techniques for wireless sensor networks:a survey. International Journal of Distributed Sensor Networks. 2013; 9(7).

[10] Izadi D, Abawajy JH, Ghanavati S, Herawan T. A data fusion method in wireless sensor networks. Sensors. 2015; 15(2):2964-79.

[11] Anisi MH, Abdullah AH, Razak SA. Energy-efficient data collection in wireless sensor networks. Wireless Sensor Network. 2011; 3(10):329-33.

[12] Upadhyay H, Mehta M. Improved APAC algorithm for minimizing delay in wireless sensor network with mobile sink. International Journal of Advanced Computer Research. 2017; 7(28):23-31.

[13] Kim KC, Kim CS. Parallel processing of sensor network data using column-oriented databases. AASRI Procedia. 2013; 5:2-8.

[14] Mota A, Oliveira LB, Rocha FF, Riserio R, Loureiro AA, Coelho CJ, et al. WISENEP: a network processor for wireless sensor networks. In proceedings of computers and communications 2006 (pp. 8-14). IEEE.

[15] Tian Y, Lv Y, Tong L. Design and application of sink node for wireless sensor network. COMPEL-The International Journal for Computation and Mathematics in Electrical and Electronic Engineering. 2013; 32(2):531-44.

[16] Rhee S, Liu S. Maximizing data reliability in wireless sensor networks. Sensor Magazine Online. 2005.

[17] Palpanas T. Real-time data analytics in sensor networks. In managing and mining sensor data 2013 (pp. 173-210). Springer.

[18] Ghayvat H, Liu J, Mukhopadhyay SC, Gui X. Wellness sensor networks: a proposal and implementation for smart home for assisted living. IEEE Sensors Journal. 2015; 15(12):7341-8.

[19] Dubey AK, Gupta U, Jain S. Analysis of k-means clustering approach on the breast cancer Wisconsin dataset. International Journal of Computer Assisted Radiology and Surgery. 2016; 11(11):2033-47.

[20] Dubey AK, Gupta U, Jain S. Comparative study of kmeans and fuzzy c-means algorithms on the breast cancer data. International Journal on Advanced
Science, Engineering and Information Technology. 2018; 8(1):18-29.

[21] Dadda A, Ouhbi I. A decision support system for renewable energy plant projects. In fifth international conference on next generation networks and services 2014 (pp. 356-62). IEEE

[22] Kolios A, Mytilinou V, Lozano-Minguez E, Salonitis $\mathrm{K}$. A comparative study of multiple-criteria decisionmaking methods under stochastic inputs. Energies. 2016; 9(7):1-21.

[23] Fishburn PC. Additive utilities with incomplete product set: applications to priorities and assignments. Operations Research. 1967; 15(3):537-42.

[24] Bridgman PW. Dimensional analysis. New Haven, CN: Yale University Process; 1992.

[25] Miller DW, Starr MK. Executive decisions and operations research. Englewood Cliffs, NJ: PrenticeHall; 1963.

[26] Kamyabpour N, Hoang DB. Modeling overall energy consumption in wireless sensor networks. arXiv preprint arXiv:1112.5800. 2011.

[27] Nagamalar T, Rangaswamy TR. Energy efficient cluster based approach for data collection in wireless sensor networks with multiple mobile sink. In international conference on industrial instrumentation and control 2015 (pp. 348-53). IEEE.

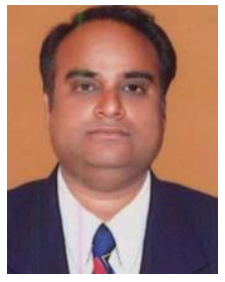

Anil Khandelwal is a Research Scholar in the department of Electronics \& Communication Engineering, Samrat Ashok Technological Institute, Vidisha, Madhya Pradesh, India. He completed his B.E. in Electronics and Telecommunication Engineering from North Maharashtra University, Jalgaon, Maharashtra in 1997 and M.Tech in Digital Communication from RGPV, Bhopal, Madhya Pradesh, India in 2010. His research interests are Wireless Sensor Network, Clustering Techniques, OFDM and MIMO.

Email: akhandelwal7@gmail.com

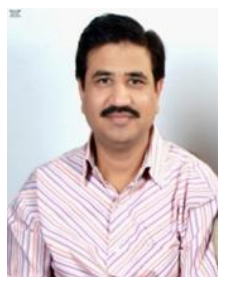

Dr. Yogendra Kumar Jain received his Ph.D. degree in Electronics \& Instrumentation Engineering from RGPV, Bhopal, Madhya Pradesh, India in 2010. He is Professor in the Department of Electronics \& Instrumentation Engineering, Samrat Ashok Technological Institute, Vidisha, Madhya Pradesh, India since 1992. He published more than 100 research paper in various International/National Journals and Conferences. He is reviewer of the IEEE Transaction on Cybernetics, Man and Systems, Elsevier Journal of Information Security and Applications, INFOCOMP Journal of Computer Science and so many conferences of IEEE, Springer and also act as the member of editorial board of reputed journals.

Email: ykjain_p@yahoo.co.in 